Check for updates

Cite this: RSC Adv., 2019, 9, 36301

Received 26th September 2019 Accepted 1st November 2019

DOI: $10.1039 / c 9 r a 07828 b$

rsc.li/rsc-advances

\section{Ultralow lattice thermal conductivity and high thermoelectric performance of monolayer KCuTe: a first principles study}

\begin{abstract}
Jinjie Gu, (D) * Lirong Huang and Shengzong Liu
Monolayer KCuTe is a new-type of two-dimensional (2D) semiconductor material with high carrier mobility and large power energy conversion efficiencies, suggesting its potential application in thermoelectric (TE) and photoelectric fields. Based on the density functional theory (DFT) and semiclassical Boltzmann transport equation, the electronic and phonon transport properties of monolayer KCuTe are systematically studied. Our results show that it possesses an ultralow lattice thermal conductivity value of nearly $\sim 0.13 \mathrm{~W} \mathrm{~m}^{-1} \mathrm{~K}^{-1}$ at $300 \mathrm{~K}$, mainly attributed to its small phonon group velocity, large Grüneisen parameters, and strong phonon-phonon scattering. Furthermore, the intralayer opposite phonon vibrations greatly restrict the heat transport. Monolayer KCuTe shows an ideal direct band gap of $\sim 1.21 \mathrm{eV}$, and a high twofold degeneracy appearing at the $\Gamma$ point gives a high Seebeck coefficient of $\sim 2070 \mu \mathrm{V} \mathrm{K} \mathrm{K}^{-1}$, leading to high TE performance. Using the transport coefficients together with constant electron relaxation time, the figure of merit $(Z T)$ can reach 2.71 at $700 \mathrm{~K}$ for the $\mathrm{p}$-type doping, which is comparable to the well-known TE material SnSe (2.6 \pm 0.3 at $935 \mathrm{~K})$. Our theoretical studies may provide perspectives to TE applications of monolayer KCUTe and stimulate further experimental synthesis.
\end{abstract}

\section{Introduction}

With the depletion of the fossil fuels, searching for renewable energy resources as well as related materials is urgently demanded. In particular, TE materials have attracted much attention, because they could convert waste heat into electric energy via the Seebeck effect. ${ }^{1,2}$ In general, the conversion efficiency of a TE material is characterized by the dimensionless figure of merit $Z T^{3}$

$$
Z T=\frac{S^{2} \sigma T}{\kappa_{\mathrm{e}}+\kappa_{1}},
$$

where $S, T, \sigma, \kappa_{\mathrm{e}}$ and $\kappa_{1}$ are the Seebeck coefficient, absolute temperature, electric conductivity, electronic and lattice thermal conductivity, respectively. Obviously, a good TE material should exhibit a larger power factor (PF) $S^{2} \sigma$ and/or low thermal conductivity. ${ }^{4}$ However, it is difficult to optimize the transport coefficients independently because of their complex competition. Some comment strategies have been studied to dispose this paradoxical relationship. For example, some common methods are optimizing electrical transport properties by band structure engineering ${ }^{5,6}$ and/or reducing a material's thermal conductivity via low dimensional technologies ${ }^{7,8}$ and

Hunan Provincial Key Laboratory of Finance \& Economics Big Data Science and Technology, School of Information Technology and Management, Hunan University of Finance and Economics, Changsha 410205, P. R. China.E-mail: gujinjie_HUFE@ 163.com phononic crystal patterning.,910 These approaches can help people to simplify these TE parameters and focus on optimizing electrical or phonon transport properties alone.

It is well known that TE materials with plicated layered configuration such as SnSe, ${ }^{11} \mathrm{PbTe}^{12}$ and $\mathrm{Bi}_{2} \mathrm{Te}_{3}$ (ref.13) have low thermal conductivity, which is one of the key factors to achieve good TE performance. Usually, the most pristine TE materials are composed of light atoms and heavy atoms, all of which possess strong anharmonicity. ${ }^{14-16}$ For instance, $\mathrm{Bi}_{2} \mathrm{O}_{2} \mathrm{Se}$ monolayer, a new star material, exhibits an intrinsically low thermal conductivity $\left(0.74 \mathrm{~W} \mathrm{~m}^{-1} \mathrm{~K}^{-1}\right.$ at $\left.300 \mathrm{~K}\right)$ due to its strong anharmonic interaction. ${ }^{17}$ For the phosphorenelike SnSe compound, its characteristic crystal structure and electronic structure lead to low $\kappa_{1}$ and high power factor. ${ }^{11}$ With the excellent electrical transport properties, the deceptively simple material SnSe monolayer shows an unexpectedly high $Z T$ of 2.76 at $700 \mathrm{~K}$ and it has become a very promising TE material. ${ }^{18}$

Very recently, the monolayer $\mathrm{KCuTe}$, as a novel layered material, has been theoretically proposed, ${ }^{19}$ which can be prepared by isovalent substitutions in layered KAgSe. The monolayer KCuTe has a favorable direct band gap with ultrahigh electron mobility of $1.3 \times 10^{4} \mathrm{~cm}^{2} \mathrm{~V}^{-1} \mathrm{~s}^{-1}$, which is not only much larger than that of the $\mathrm{MoS}_{2}$ monolayer ${ }^{20}$ but also can be on a par with that of black phosphorene (1.0-2.6 $\times$ $\left.10^{4} \mathrm{~cm}^{2} \mathrm{~V}^{-1} \mathrm{~s}^{-1}\right) .{ }^{21}$ Further, its excellent electrical properties, large power energy conversion efficiencies $(\sim 21.5 \%)$ and applicable band gap, offer multiple perspectives for 
applications in photoelectric and photovoltaic solar cells. ${ }^{\mathbf{1 9}}$ Considering these outstanding performances, it is necessary to study comprehensively on its TE properties. Based on theoretical calculations, we systematically study the TE properties of monolayer KCuTe. Meanwhile, we find that it exhibits a high Seebeck coefficient of $2070 \mu \mathrm{V} \mathrm{K} \mathrm{K}^{-1}$ and an ultralow lattice thermal conductivity of $0.13 \mathrm{~W} \mathrm{~m}^{-1} \mathrm{~K}^{-1}$, which are prerequisites for achieving high TE performance. Detailed discussions of phonon spectrums, atomic vibration modes, phonon group velocity, and Grüneisen parameters are presented to explain its heat transport ability. The maximum $Z T$ value is as high as 2.71 at $700 \mathrm{~K}$ for p-type doping. It is revealed that the monolayer KCuTe possesses extraordinary TE response.

\section{Computational methods}

Within the framework of DFT, the electronic properties of monolayer KCuTe were investigated by using the projector augmented wave method, which was implemented in the Vienna $a b$ initio Simulation Package (VASP). ${ }^{22}$ The generalized gradient approximation (GGA) ${ }^{23}$ in the Perdew-Burke-Ernzerhof $(\mathrm{PBE})^{\mathbf{2 4}}$ was employed. The cutoff energy of the plane wave was set as $500 \mathrm{eV}$ and $11 \times 11 \times 1$ Monkhorst-Pack $k$-mesh were adopted in the calculations. A vacuum region of $25 \AA$ was set along the $z$ direction to avoid the interactions between periodic layers. The semi-empirical DFT-D3 (ref. 25) correction was included to evaluate the van der Waals interactions. The atomic positions are fully relaxed with the Hellman-Feynman force $0.01 \mathrm{eV} \AA^{-1}$, while the total energy convergence criteria was $10^{-5} \mathrm{eV} \AA^{-1}$. The optimized lattice parameter of monolayer KCuTe is $a=4.26 \AA$, which is consistent with the previous theoretical prediction. ${ }^{19}$ In order to calculate its electronic structure, the hybrid functional approach of Heyd-ScuseriaErnzerhof (HSE06) was employed. ${ }^{26}$ By using the Boltzmann transport theory and the rigid band approach, the electronic transport properties were obtained, implemented in the BoltzTraP code. ${ }^{27}$ The constant relaxation time approximation was employed, which was valid because the relaxation time doesn't vary strongly within the energy scale of $k_{\mathrm{B}} T \cdot{ }^{28}$ Such method has accurately assessed TE properties of many materials. ${ }^{29}$ To accurately perform Fourier interpolation of the Kohn-Sham eigenvalues, a dense $45 \times 45 \times 1 k$-mesh was employed in the Brillouin zone (BZ).

Using the ShengBTE code,$^{30}$ the phonon transport properties were evaluated. The harmonic second-order interaction force constants (2nd IFCs) and phonon spectrum were obtained by the VASP and Phonopy packages ${ }^{31}$ using $5 \times 5 \times 1$ supercell with $3 \times 3 \times 1 k$-mesh. The anharmonic third-order IFCs (3rd IFCs) were obtained using the finite-difference method, ${ }^{32}$ where the influence of the seventh nearest neighbors is considered. Additionally, the convergence of the $\kappa_{1}$ as a function of the $k$ mesh was carefully tested. A dense $35 \times 35 \times 1 k$-mesh was employed for the calculation of the lattice thermal conductivity. In order to convert conductance of monolayer material to conductivity, the effective thickness of $7.8 \AA$ was chosen in monolayer KCuTe.

\section{Results and discussions}

\subsection{Electronic structures}

The monolayer KCuTe shows an orthorhombic structure with the space group P4/nmm symmetry (129), as shown in Fig. 1(a) and (b). We can see clearly that the monolayer KCuTe exhibits five atomic-layers in the form of $\mathrm{K}-\mathrm{Cu}-\mathrm{Te}-\mathrm{Cu}-\mathrm{K}$ and six atoms per unit cell, which greatly increases the complexity of the structure. Moreover, its conspicuously plicated configuration can give rise to strong lattice vibrations, greatly suppressing phonon transport ability. ${ }^{17}$

The band structures and corresponding projected density of state (DOS) of monolayer KCuTe along a series of high symmetry points in the BZ are shown in Fig. 1(c). It has direct band gap with the valence band maximum (VBM) and conduction band minimum (CBM) locating at $\Gamma$ point. In the vicinity of Fermi level, the conduction bands (CB) are rarely occupied by the $\mathrm{K}, \mathrm{Cu}$ and $\mathrm{Te}$ atoms, whereas the valence bands (VB) are primarily contributed by the $\mathrm{Cu}$ and $\mathrm{Te}$ atoms. Interestingly, a twofold degeneracy could be observed at $\Gamma$ point. It is reported that band degeneracy has been proved to be critical for achieving high TE performance ${ }^{33,34}$ and can be realized through proper band engineering. ${ }^{35}$ In addition, this degenerated electronic state is relatively flat at the VB edge, which is in favour of high $\mathrm{PF}^{36}$ Several sharp peaks are observed at VB in DOS, a signal of a dramatic increase in Seebeck coefficients. ${ }^{37,38}$ Therefore, unique DOS structure and an intrinsic VB degeneracy appear together in the monolayer KCuTe, which are good electronic features for highperformance of TE equipments.

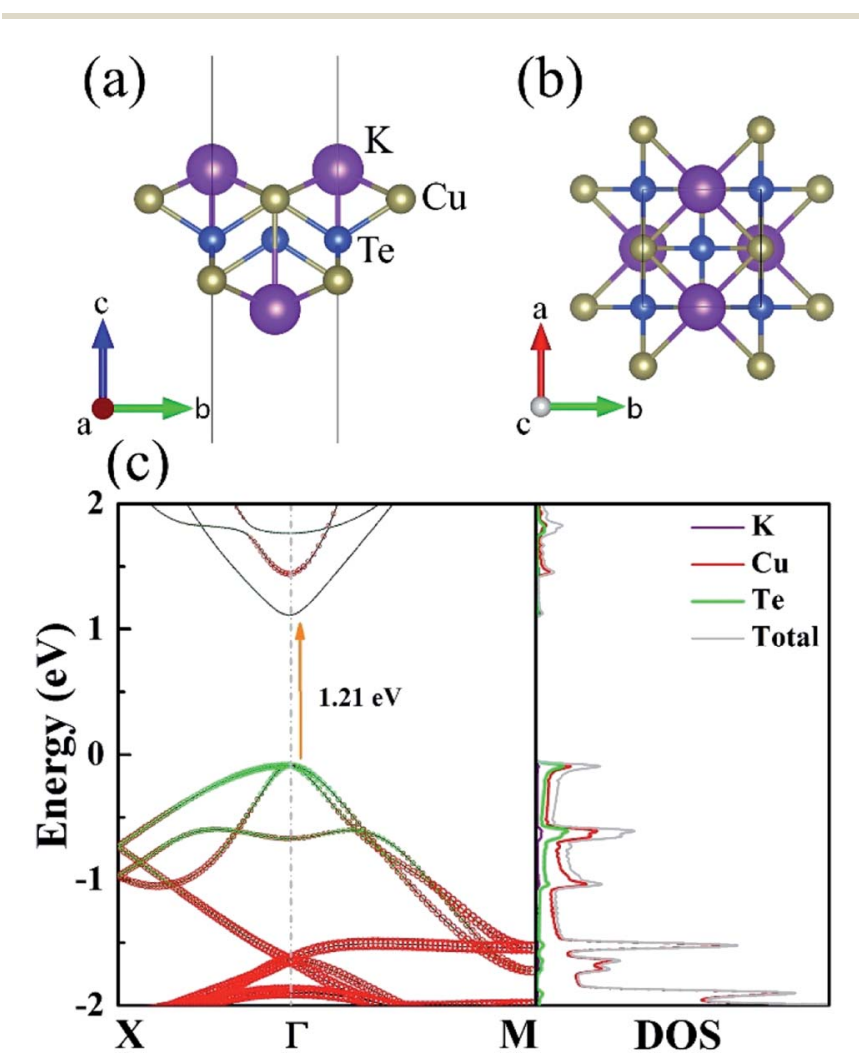

Fig. 1 Side (a) and top (b) views of the atomic structure of monolayer KCuTe. (c) The band structures and the corresponding DOS. 


\subsection{Electron transport properties}

With the constant relaxation time assumption, the electronic transport properties for KCuTe are obtained. Here, we computed the Seebeck coefficients $S$, electrical conductivity $\kappa_{1}$, and electronic thermal conductivity $\kappa_{\mathrm{e}}$, and $\mathrm{PF} S^{2} \sigma$ of monolayer KCuTe under the temperature from 300 to $700 \mathrm{~K}$. Calculative results are shown in Fig. 2(a)-(d) as a function of chemical potential $\mu$, of which the positive and negative side correspond to n-type and p-type doping, respectively. By using the Boltzmann transport theory, the $S$ and $\sigma$ could be expressed as $^{27}$

$$
\begin{gathered}
S_{\alpha \beta}(T, \mu)=\frac{1}{e T V \sigma_{\alpha \beta}(T, \mu)} \int \sum_{\alpha \beta}(\varepsilon)(\varepsilon-u)\left[-\frac{\partial f_{\mu}(T, \varepsilon)}{\partial \varepsilon}\right] \mathrm{d} \varepsilon, \\
\sigma_{\alpha \beta}(T, \mu)=\frac{1}{V} \int \sum_{\alpha \beta}(\varepsilon)\left[-\frac{\partial f_{\mu}(T, \varepsilon)}{\partial \varepsilon}\right] \mathrm{d} \varepsilon \\
\sum_{\alpha \beta}(\varepsilon)=\frac{e^{2}}{N_{0}} \sum_{i, k} \tau v_{\alpha}(i, \boldsymbol{k}) v_{\beta}(i, \boldsymbol{k}) \frac{\delta\left(\varepsilon-\varepsilon_{i, k}\right)}{\mathrm{d} \varepsilon}
\end{gathered}
$$

where $\alpha$ and $\beta$ are Cartesian indices, $V$ is the volume of the unit cell and $\sum_{\alpha \beta}(\varepsilon)$ is the transport distribution function. As shown in Fig. 2(a), the monolayer KCuTe exhibits relatively large values of the Seebeck coefficients. At room temperature, the Seebeck coefficients can reach to 2070 and $1970 \mu \mathrm{V} \mathrm{K}{ }^{-1}$ for $\mathrm{p}$ - and n-type doping. Meanwhile, we can also find that the Seebeck coefficient for n-type doping is slightly larger than that of the p-type doping, which can attribute to its DOS. As has been reported, ${ }^{35}$ the multiple degeneracy usually significantly improve the Seebeck coefficient. We can clearly find that the Seebeck coefficients exhibit the obvious temperature-dependent behavior. At low chemical potentials, the Seebeck coefficients appear peaks due to the sharp energy dependence of the total DOS, i.e., large $\partial n(\varepsilon) / \partial(\varepsilon)$, as shown in Fig. 1. For doping system, the Seebeck coefficients could be derived according to the Mott relation ${ }^{39}$

$$
S=\frac{\pi^{2} k_{\mathrm{B}}^{2} T}{3 e}\left\{\frac{1}{n} \frac{\mathrm{d} n(\varepsilon)}{\mathrm{d} \varepsilon}+\frac{1}{\mu} \frac{\mathrm{d} n(\varepsilon)}{\mathrm{d} \varepsilon}\right\} \varepsilon=\mu
$$

We can find that the Seebeck coefficient can be further enhanced by optimizing the carrier concentration.

The electrical conductivity $\sigma / \tau$ as a function of chemical potential is shown in Fig. 2(b). In fact, theoretical calculations prove that the p-type doping shows larger $\sigma / \tau$ than that of the ntype ones. The slopes of the $\sigma / \tau$ around the VBM and CBM is flatted by increasing temperature because the electrons obey the Fermi-Dirac distribution.

Besides, the electronic thermal conductivity $\kappa_{\mathrm{e}}$ can be calculated by using the Wiedemann-Franz law: ${ }^{\mathbf{4 0}}$

$$
\kappa_{\mathrm{e}}=L \sigma T
$$

(a)
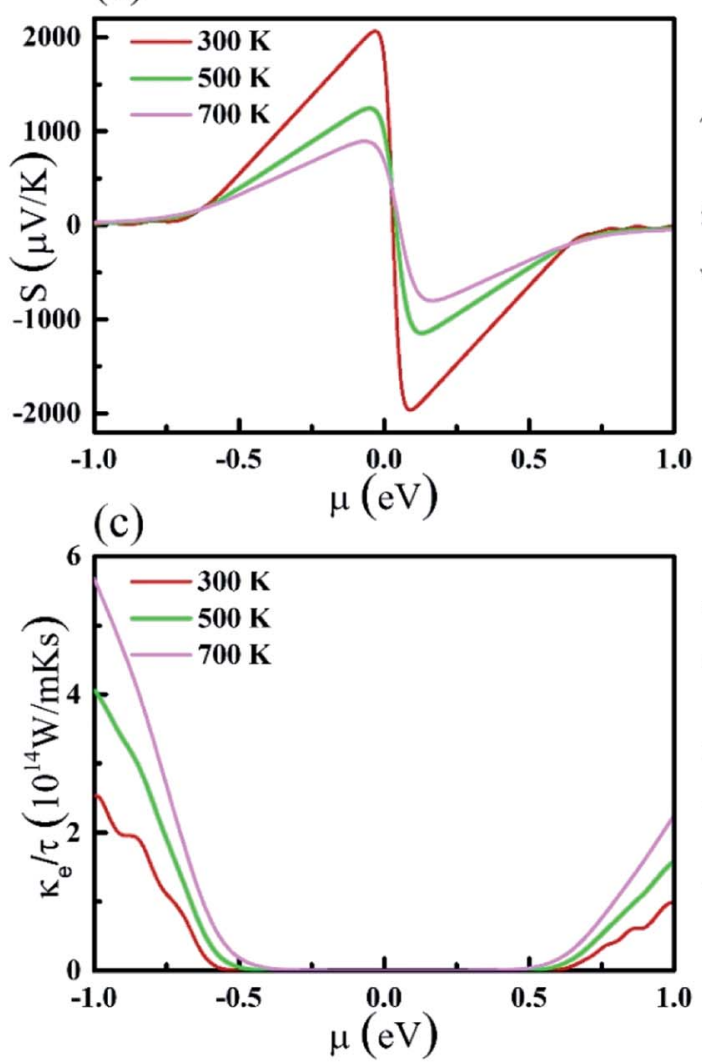

(b)

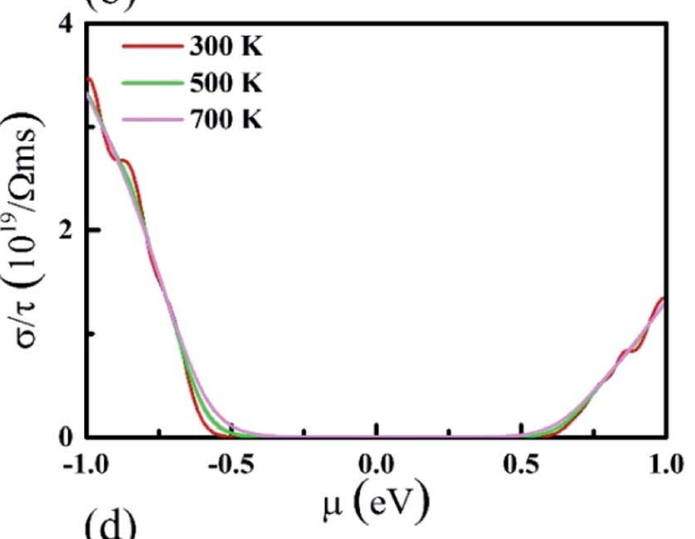

(d)

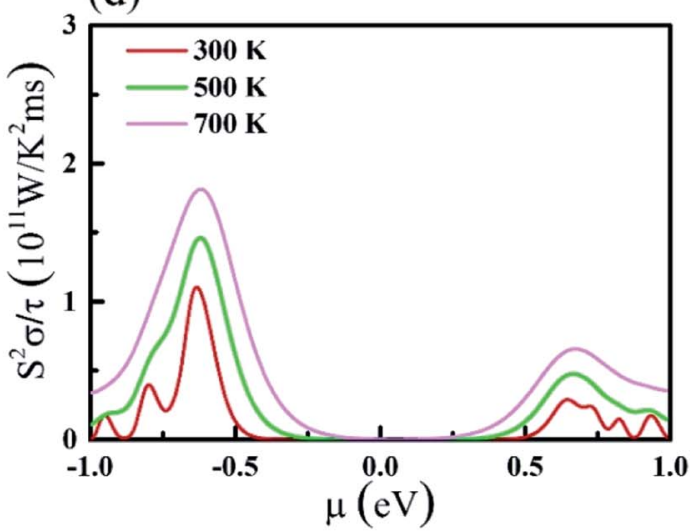

Fig. 2 (a) Seebeck coefficients, (b) electrical conductivity, (c) electronic thermal conductivity, and (d) power factor with respect to the scattering time at different temperatures $(300,500$, and $700 \mathrm{~K})$ as functions of the chemical potential. 
where $L=\pi^{2} k_{\mathrm{B}}{ }^{2} / 3 e^{2}$ is the Lorenz number. We can clearly see that the $\kappa_{\mathrm{e}}$ and $\sigma$ exhibit similar behaviors since both of them are originated from the electronic structure. Combining with the Seebeck coefficient and the electrical conductivity, we calculate the $\mathrm{PF}$ with respect to the scattering time as shown in Fig. 2(d). The PF of p-type doping is visibly larger than that of ntype doping for monolayer KCuTe, owing to its high $\sigma$ in p-type doping. Additionally, the maximum peak PF of $1.8 \times 10^{11} \mathrm{~W} \mathrm{~K}^{-2}$ $\mathrm{ms}^{-1}$ is due to its ideal DOS at the VB edge. The twofold degeneracy which can rise large number of carriers and result in high Seebeck coefficients is another reason of high PF.

\subsection{Phonon transport properties}

The phonon dispersions and partial atomic phonon density of states (PhDOS) of monolayer KCuTe are shown in Fig. 3(a). Since the acoustic branches play a major contribution to heat transport, ${ }^{41}$ we highlight these phonon modes with different colors. Here, the negative phonon frequency is not observed, demonstrating its dynamical stability. Surprising, we can find that it shows a very narrow phonon frequency interval with the maximum frequency of the optical mode about 4.5 THz. Such low phonon frequencies are beneficial to low thermal conductivity. Additionally, its lowest optical mode frequency is as low as $0.39 \mathrm{THz}$, which is much lower than that of the phosphorene like SnSe (0.99 THz). ${ }^{42}$ We can also find that the lowest optical modes are overlapping with its acoustic branches, resulting in strong acoustic-optical interactions and low lattice thermal conductivity. From the corresponding PhDOS, we can see that the phonon branches are evenly occupied by $\mathrm{K}, \mathrm{Cu}$ and Te atoms in the full-frequency region, which further verify its stability. ${ }^{43}$ This phenomenon can attribute to the strongly overlapping electron states. The out-of-plan acoustic mode (ZA) has parabolic dispersion, which is a unique character for atomically thin materials. We can note that these flat phonon modes and protuberant PhDOS can give rise to small phonon velocities.

To further study each modes phonon spectrum, some typical vibration modes of KCuTe sheet at $\Gamma$ point are shown in
Fig. 3(b). One can see clearly that the vibrations of the acoustic modes are strictly along the in-plane (LA and TA) or out-of-plane (TA), which are an inherent vibration property. For the lowest optical branch, we find that the upper $\mathrm{Cu}$ atoms move in opposite directions with respect to the lower $\mathrm{Cu}$ atoms, including both intralayer Se atoms, while the K atoms keep still. Such atomic vibration mode will greatly suppress heat transport ability in layered materials. Similar with graphene, ${ }^{\mathbf{4 4}}$ these optical modes are easily thermally activated at room temperature, leading to low thermal conductivity via enhancing phonon-phonon scattering. ${ }^{45}$

From the phonon kinetic theory, the lattice thermal conductivity in a solid is defined by

$$
\kappa_{1}=\frac{1}{V} \sum_{\lambda} C_{\lambda} v_{\lambda}^{2} \tau_{\lambda}
$$

where $C_{\lambda}$ is mode heat capacity, $v_{\lambda}$ is the phonon group velocity, and the $\tau_{\lambda}$ is the phonon relaxation time, respectively. To investigate the thermal transport properties of KCuTe sheet, the $\kappa_{1}$, at temperature from 200 to $800 \mathrm{~K}$, is shown in Fig. 4(a). In many structures, ${ }^{\mathbf{1 2 , 4 6}}$ the $\kappa_{1}$ exhibits decreasing behavior monotonously with the temperature increasing. Specifically, in low temperature region, the $\kappa_{1}$ decrease rapidly. Furthermore, it also shows an ultralow $\kappa_{1}$ of $0.13 \mathrm{~W} \mathrm{~m}^{-1} \mathrm{~K}^{-1}$ at $300 \mathrm{~K}$, which is lower than that of the KAgTe $\left(0.22 \mathrm{~W} \mathrm{~m}^{-1} \mathrm{~K}^{-1}\right) .{ }^{47}$ Such low $\kappa_{\mathrm{l}}$ indicates it might be possesses excellent TE response.

The phonon velocity is a very important physical parameter for the calculation of thermal conductivity. It can be obtained by using the phonon spectrum,

$$
v_{\lambda, \boldsymbol{q}}=\frac{\partial \omega_{\lambda, \boldsymbol{q}}}{\partial \boldsymbol{q}},
$$

where $\omega_{\lambda, q}$ is the phonon frequency, and plot them in Fig. 4(b). Obviously, we can see that the KCuTe sheet exhibits very small phonon velocities. For its acoustic branches (ZA, TA, and LA), the average phonon velocities are $1.05,0.82$, and $0.83 \mathrm{~km} \mathrm{~s}^{-1}$, respectively. Compared with the monolayer $\mathrm{PbSe},{ }^{41}$ the phonon velocities of the KCuTe exhibits smaller phonon velocities, which can attribute to its flat phonon dispersions. Based on the (a)

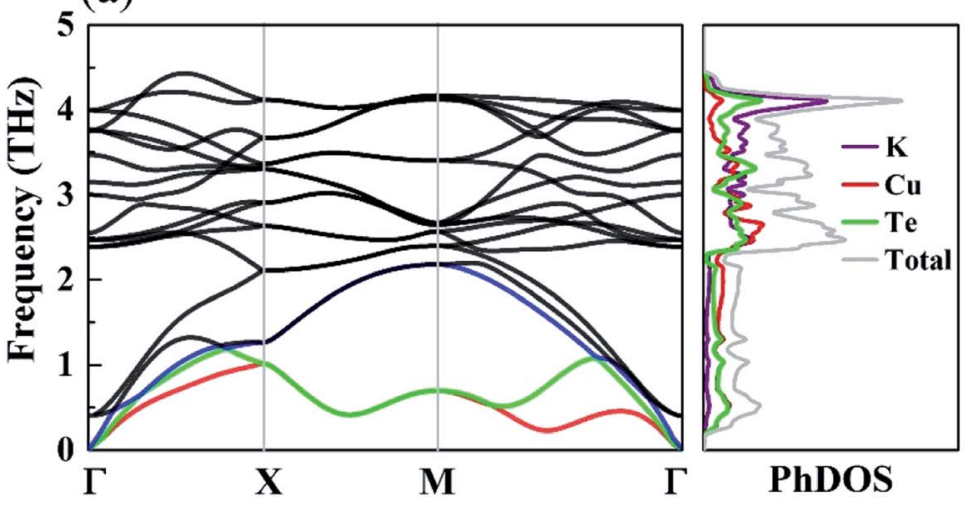

(b)

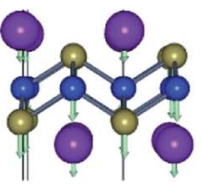

$\mathrm{ZA}$

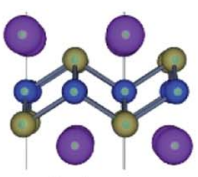

LA

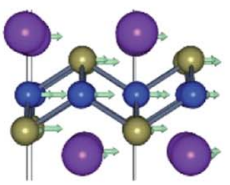

TA

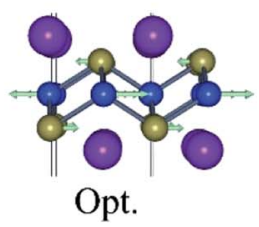

Fig. 3 (a) Phonon dispersion and total and partial phonon density of states (PhDOS) of monolayer KCuTe. (b) The corresponding vibrational modes of the acoustic phonon branches (the red, green and blue lines corresponding to the ZA, TA, and LA) and the lowest optical branch (Opt.) near and at $\Gamma$ point. 
(a)

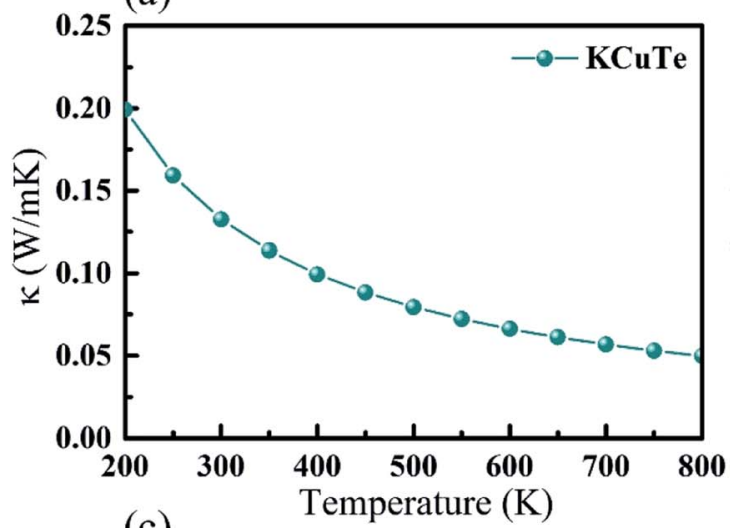

(c)

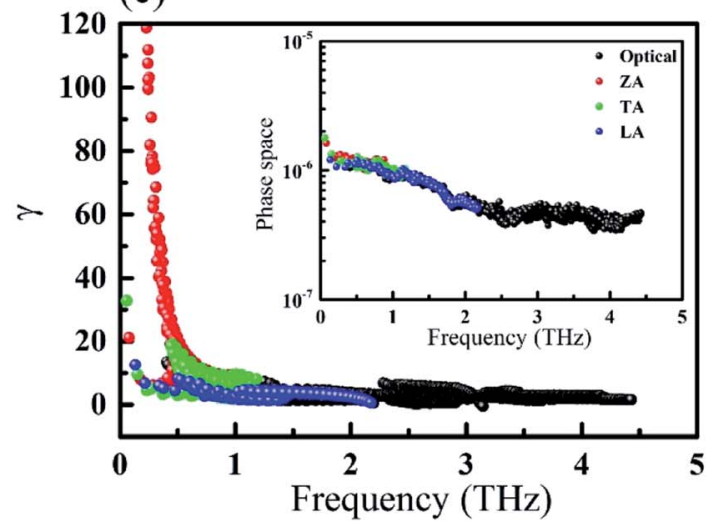

(b)

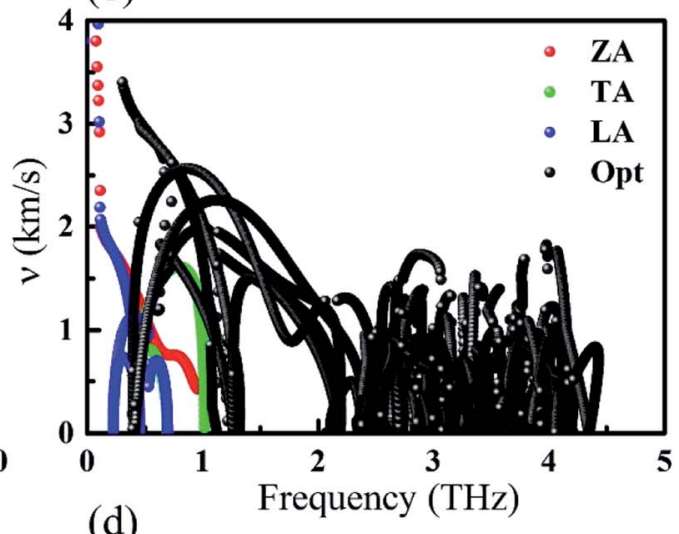

(d)

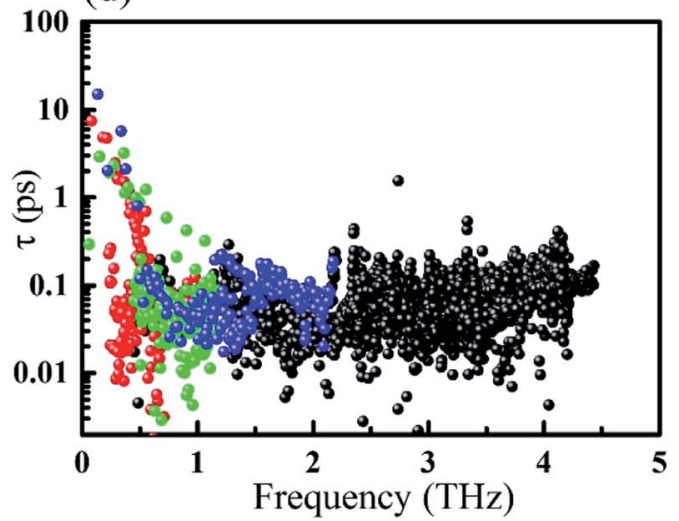

Fig. 4 (a) Calculated lattice thermal conductivity with respect to temperature for monolayer KCuTe. (b) Phonon group velocity, (c) Grüneisen parameters, and (d) phonon relaxation time at $300 \mathrm{~K}$. The inset of (c) is three phonon phase space.

eqn (6), we can conclude that these small phonon velocities lead to low thermal conductivity.

Besides the phonon velocities, we further study its Grüneisen parameters and phonon relaxation time of each phonon modes, as shown in Fig. 4(c) and (d). The Grüneisen parameters can qualitatively describe the anharmonic interactions of a crystal. In general, it can be computed from the relationship between phonon frequency and volume change as below:

$$
\gamma_{(\lambda, \boldsymbol{q})}=-\frac{V}{\omega_{\lambda, \boldsymbol{q}}} \frac{\partial \omega_{\lambda, \boldsymbol{q}}}{\partial V}
$$

Usually, a large $|\gamma|$ means that it might have a strong phonon-phonon anharmonic scattering. Surprisingly, we can see clearly that the monolayer KCuTe exhibits very large Grüneisen parameters, indicating that it possesses strong anharmonicity. From the Fig. 4(c), we find that the Grüneisen parameter of ZA mode is much larger than that of the TA and LA mode. Herein, the phonon relaxation time can be obtained by the summation of various scattering processes. ${ }^{48}$ Compared with some common TE materials, ${ }^{17,41}$ we can see clearly that it shows a very short relaxation time (nearby $0.1 \mathrm{ps}$ ), leading to low lattice thermal conductivity. To further analyze its phonon (a)

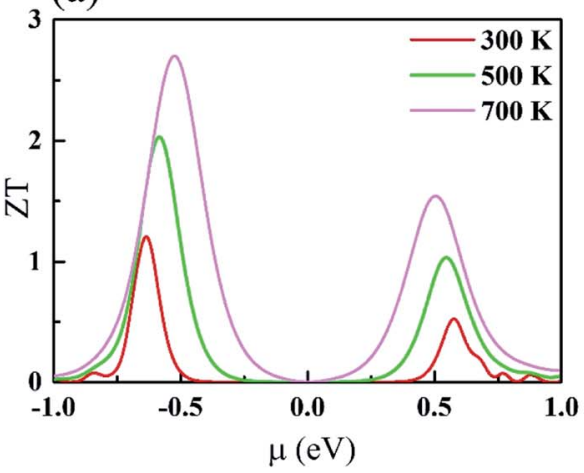

(b)

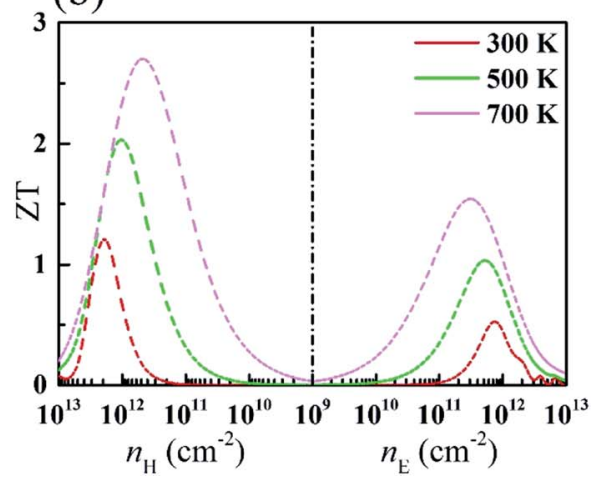

Fig. $5 Z T$ with respect to (a) chemical potential and (b) carrier concentration for monolayer KCuTe. 
relaxation time, the three phonon scattering phase space (P3) is presented as shown in the insert of Fig. 4(c). One can see that the monolayer KCuTe possesses a relatively larger P3 in the fullfrequency region, which indicates that it exists strong phononphonon scattering and greatly suppress the phonon transport.

\subsection{Thermoelectric figure of merit}

Using the electronic and phonon transport coefficients available, we have computed the TE properties of monolayer KCuTe. Herein, the electronic scattering time $\tau$ is $3.5 \mathrm{fs}$, extracted from previous report. ${ }^{49}$ The $Z T$ values with respect to chemical potential and carrier concentration at 300, 500 and $700 \mathrm{~K}$ are shown in Fig. 5(a) and (b). Additionally, we can see that the ZT of the p-type doping is higher that of the n-type doping, which can attribute to the difference of PF. With the temperature increasing, the peak becomes wider and the peak position moves toward $\mu=0$. We can see that the $Z T$ values can approach 2.71 and 1.51 for p-type and n-type doping at $700 \mathrm{~K}$ since it has a lower thermal conductivity. Moreover, the corresponding concentration of maximum $Z T$ is about $5 \times 10^{11} \mathrm{~cm}^{-2}$ for p-type doping.

\section{Conclusion}

In summary, using DFT and Boltzmann transport equation, we systematically studied the electronic and phonon transport properties of monolayer KCuTe. Results show that it possesses an ideal band gad $(1.21 \mathrm{eV})$ with a high Seebeck coefficients of $2070 \mu \mathrm{V} \mathrm{K}^{-1}$ at room temperature. Since the multiple valley degeneracy around VB, it exhibits outstanding electrical transport properties. Additionally, its unique crystal texture, small phonon velocity, large Grüneisen parameters, and short phonon relaxation time greatly suppress the heat transport ability, leading to ultralow lattice thermal conductivity of $0.13 \mathrm{~W}$ $\mathrm{m}^{-1} \mathrm{~K}^{-1}$ at $300 \mathrm{~K}$. The $Z T$ of monolayer KCuTe is as high as 2.71 at $700 \mathrm{~K}$ for p-type doping. Collectively, our works demonstrate that the monolayer KCuTe possesses excellent TE performance and will become a hopeful candidate in TE material field.

\section{Conflicts of interest}

There are no conflicts to declare.

\section{Acknowledgements}

This work was supported by the Research project of Education Department of Hunan Province (No. 18A441, 18C0958), and the Open Research Fund of Hunan Provincial Key Laboratory of Network Investigational Technology (No. 2017WLZC006), and the Key Project of Educational Planning of Hunan Province (No. XJK18DJA1).

\section{References}

1 S. Chu, Y. Cui and N. Liu, Nat. Mater., 2017, 16, 16-22. 2 M. Aneke and M. Wang, Appl. Energy, 2016, 179, 350-377.
3 J. Yang, L. Xi, W. Qiu, L. Wu, X. Shi, L. Chen, J. Yang, W. Zhang, C. Uher and D. J. Singh, npj Comput. Mater., 2016, 2, 15015.

4 J. M. Skelton, S. C. Parker, A. Togo, I. Tanaka and A. Walsh, Phys. Rev. B: Condens. Matter Mater. Phys., 2014, 89, 205203.

5 Z. Wang, L. Zhao, K. F. Mak and J. Shan, Nano Lett., 2017, 17, 740-760.

6 L.-P. Tang, L.-M. Tang, H. Geng, Y.-P. Yi, Z. Wei, K.-Q. Chen and H.-X. Deng, Appl. Phys. Lett., 2018, 112, 012101.

7 D. Li, J. He, G. Ding, Q. Tang, Y. Ying, J. He, C. Zhong, Y. Liu, C. Feng, Q. Sun, et al., Adv. Funct. Mater., 2018, 28, 1801685.

8 Y.-Y. Liu, Y.-J. Zeng, P.-Z. Jia, X.-H. Cao, X. Jiang and K.-Q. Chen, J. Phys.: Condens. Matter, 2018, 30, 275701.

9 X. Y. Mi, X. Yu, K. L. Yao, X. Huang, N. Yang and J. T. Lü, Nano Lett., 2015, 15, 5229-5234.

10 G. Xie, D. Ding and G. Zhang, Adv. Phys.: X, 2018, 3, 1480417. 11 L. D. Zhao, S. H. Lo, Y. Zhang, H. Sun, G. Tan, C. Uher, C. Wolverton, V. P. Dravid and M. G. Kanatzidis, Nature, 2014, 508, 373-377.

12 J. P. Heremans, V. Jovovic, E. S. Toberer, A. Saramat, K. Kurosaki, A. Charoenphakdee, S. Yamanaka and G. J. Snyder, Science, 2008, 321, 554-557.

13 M. Scheele, N. Oeschler, K. Meier, A. Kornowski, C. Klinke and H. Weller, Adv. Funct. Mater., 2009, 19, 3476-3483.

14 G. Xie, Z. Ju, K. Zhou, X. Wei, Z. Guo, Y. Cai and G. Zhang, npj Comput. Mater., 2018, 4, 21.

15 X. Yu, C. Cheng, Y. Pei, W. Di, K. Peng, X. Zhou, S. Gong, J. He, Y. Zhang and Z. Zhi, Phys. Rev. B: Condens. Matter Mater. Phys., 2016, 94, 125203.

16 C. W. Li, O. Hellman, J. Ma, A. F. May, H. B. Cao, X. Chen, A. D. Christianson, G. Ehlers, D. J. Singh and B. C. Sales, Phys. Rev. Lett., 2014, 112, 175501.

17 X.-L. Zhu, P.-F. Liu, G. Xie and B.-T. Wang, Phys. Chem. Chem. Phys., 2019, 21, 10931-10938.

18 F. Q. Wang, S. Zhang, J. Yu and Q. Wang, Nanoscale, 2015, 7, 15962-15970.

19 W. Xu, R. Wang, B. Zheng, X. Wu and H. Xu, ACS Appl. Mater. Interfaces, 2019, 11, 14457-14462.

20 S. Kim, A. Konar, W.-S. Hwang, J. H. Lee, J. Lee, J. Yang, C. Jung, H. Kim, J.-B. Yoo, J.-Y. Choi, et al., Nat. Commun., 2012, 3, 1011.

21 J. Qiao, X. Kong, Z.-X. Hu, F. Yang and W. Ji, Nat. Commun., $2014,5,4475$.

22 G. Kresse and J. Furthmüller, Phys. Rev. B: Condens. Matter Mater. Phys., 1996, 54, 11169.

23 P. E. Blöchl, Phys. Rev. B: Condens. Matter Mater. Phys., 1994, 50, 17953.

24 G. Kresse and D. Joubert, Phys. Rev. B: Condens. Matter Mater. Phys., 1999, 59, 1758-1775.

25 N. Marom, A. Tkatchenko, M. Rossi, V. V. Gobre, O. Hod, M. Scheffler and L. Kronik, J. Chem. Theory Comput., 2011, 7, 3944-3951.

26 J. Heyd, G. E. Scuseria and M. Ernzerhof, J. Chem. Phys., 2003, 118, 8207-8215.

27 G. K. H. Madsen and D. J. Singh, Comput. Phys. Commun., 2006, 175, 67-71. 
28 L. Chaput, P. Pécheur and C. Scherrer, Phys. Rev. B: Condens. Matter Mater. Phys., 2007, 75, 045116.

29 D. Parker and D. J. Singh, Phys. Rev. B: Condens. Matter Mater. Phys., 2010, 82, 035204.

30 W. Li, J. Carrete, N. A. Katcho and N. Mingo, Comput. Phys. Commun., 2014, 185, 1747-1758.

31 A. Togo, F. Oba and I. Tanaka, Phys. Rev. B: Condens. Matter Mater. Phys., 2008, 78, 134106.

32 W. Li, L. Lindsay, D. A. Broido, D. A. Stewart and N. Mingo, Phys. Rev. B: Condens. Matter Mater. Phys., 2012, 86, 174307.

33 L. D. Zhao, V. P. Dravid and M. G. Kanatzidis, Energy Environ. Sci., 2014, 7, 251-268.

34 X. L. Zhu, P. F. Liu, G. Xie, W. X. Zhou, B. T. Wang and G. Zhang, Nanomaterials, 2019, 9, 597.

35 Z. M. Gibbs, F. Ricci, G. Li, H. Zhu, K. Persson, G. Ceder, G. Hautier, A. Jain and G. J. Snyder, npj Comput. Mater., 2017, 3, 8 .

36 C. Fu, H. Wu, Y. Liu, J. He, X. Zhao and T. Zhu, Adv. Sci., 2016, 3, 1600035.

37 H. Peng, N. Kioussis and G. J. Snyder, Phys. Rev. B: Condens. Matter Mater. Phys., 2014, 89, 195206.
38 G. Ding, C. Wang, G. Gao, K. Yao, C. Dun, C. Feng, D. Li and G. Zhang, Nanoscale, 2018, 10, 7077-7084.

39 J. P. Heremans, Acta Phys. Pol., A, 2005, 108, 609-634.

40 M. Jonson and G. Mahan, Phys. Rev. B: Condens. Matter Mater. Phys., 1980, 21, 4223.

41 P.-F. Liu, T. Bo, J. Xu, W. Yin, J. Zhang, F. Wang, O. Eriksson and B.-T. Wang, Phys. Rev. B, 2018, 98, 235426.

42 C. W. Li, J. Hong, A. F. May, D. Bansal, S. Chi, T. Hong, G. Ehlers and O. Delaire, Nat. Phys., 2015, 11, 1063.

43 T. Ouyang, E. Jiang, C. Tang, J. Li, C. He and J. Zhong, J. Mater. Chem. A, 2018, 6, 21532-21541.

44 C. H. Lui and T. F. Heinz, Phys. Rev. B: Condens. Matter Mater. Phys., 2013, 87, 121404.

45 J. Ding, B. Xu, Y. Lin, C. Nan and W. Liu, New J. Phys., 2015, 17, 083012.

46 G. Xie, Y. Guo, X. Wei, K. Zhang, L. Sun, J. Zhong, G. Zhang and Y. W. Zhang, Appl. Phys. Lett., 2014, 104, 233901.

47 M. M. Mahmoud, E. K. Rugut, M. P. Molepo and D. P. Joubert, Eur. Phys. J. B, 2019, 92, 87.

48 K. Hess, Appl. Phys. Lett., 1979, 35, 484-486.

49 A. Parveen and G. Vaitheeswaran, Sci. Rep., 2018, 8, 13071. 